Génét. Sél. Evol., 1984, 16 (2), 185-194

\title{
Effectif et taux de masculinité des groupes de chats dans les familles humaines (région lyonnaise)
}

\author{
J.-M. LEGAY et D. CHESSEL \\ Laboratoire de Biométrie, Université Cl. Bernard (Lyon I) \\ 43, boulevard du 11-Novembre-1918, F 69622 Villeurbanne Cedex
}

\begin{abstract}
Résumé
L'analyse statistique de la distribution des chats dans un échantillon de 1258 familles humaines nous permet d'aborder le problème de la structure des groupes et de leur taux de masculinité.

La répartition des chats mâles dans les familles humaines suit une loi de Poisson, celle des femelles une binômiale négative ; mais la distribution conjointe est plus complexe et l'analyse du taux de masculinité apparaît donc comme justifiée. On peut montrer que le taux de masculinité diffère de un demi, dépend de la taille du groupe et décroît significativement quand celle-ci augmente. L'interprétation est discutée.
\end{abstract}

Mots clés : Chats, population, structure.

\section{Summary}

Size and sex-ratio of cat groups in human families (Lyon area)

The problems of group structure and sex-ratio are studied by statistical analysis of the distribution of cats in a sample of 1258 human families. The repartition of male cats in human families approximates to a Poisson distribution and that of females to a negative binomial. On the other hand the combined distribution is more complex and therefore an analysis of sex-ratio was performed. This showed that the sex-ratio differs from 0.5 , is not independent of size of group and decreases significantly as group size is larger. The interpretation is discussed.

Key words : Cats, populations, structure.

\section{Introduction}

Si la structure génétique des populations de chats a commencé d'être étudiée (par exemple : Dreux, 1967, 1978 ; Searle, 1949 ; Robinson, 1959, 1969) et mérite d'amples développements, la biologie de ces populations est encore bien mal connue (ToDd, 1977). Ainsi, FAGEN (1978) pratique une enquête téléphonique dans la ville d'Urbana (Illinois) et consulte 836 familles, mais ne réunit qu'un petit échantillon 
de 124 chats et n'en interprète pas la distribution. Un certain nombre d'auteurs d'autre part s'intéresse aux populations insulaires de chats dans la mesure où elles seraient, dans quelques cas favorables, peu ou pas influencées par l'homme : îles Kerguelen (Pascal, 1980), île aux Cochons (Derenne \& Mougrn, 1976), île Macquarie (Jones, 1977), île Marion (VAN AARde, 1978). L'étude la plus documentée est sans doute celle de Van Aarde qui réunit un échantillon important de 825 chats, dont plus de 90 p. 100 vivent isolés; il examine les groupes disponibles et constate les modifications de leur effectif et de leur structure en fonction de la saison (les modalités de la reproduction étant par ailleurs analysées).

En fait, les chats sont considérés comme des animaux domestiques dans la mesure où ils vivent avec les hommes, mais ils ont gardé jusqu'à présent une très large autonomie de reproduction, et l'on peut penser que leurs populations ont conservé un certain nombre de caractéristiques originelles. C'est d'ailleurs surtout leur comportement social qui a attiré l'attention et plus récemment leurs structures de socialité.

Dans cet article, nous nous intéressons aux populations ordinaires de chats domestiques et souhaitons décrire et discuter la distribution des chats dans les familles humaines. Nous verrons les structures des groupes (effectif et taux de masculinité) qui en résultent.

\section{Matériel et méthodes}

La plupart des renseignements concernant la biologie des chats proviennent des hommes auxquels ils sont attachés. Nous avons procédé par voie d'enquêtes orales; nous sommes passés par l'intermédiaire d'étudiants et d'enseignants sous le contrôle desquels les données ont été recueillies. En partant des établissements scolaires, nous avons pratiqué neuf sondages pendant l'hiver 1979-1980, qui couvrent assez bien, dans la région lyonnaise (Rhône surtout, Isère, Ain, Saône et Loire secondairement), la diversité des familles humaines tant du point de vue de leur localisation, urbaine ou rurale, que de leur position socio-professionnelle. Les élèves (et par suite les familles) des classes ou groupes choisis ont tous été interrogés. En réponse aux quatre questions posées (nombre de chats, sexe, âge, fécondité), les ignorances ou imprécisions relevaient parfois du manque d'information sur l'âge (mais pas spécialement chez les jeunes) ou la fécondité des femelles, mais pratiquement jamais sur le sexe. Dans ces conditions nous disposons de données utilisables provenant de 1258 familles.

Pour chacune de celles-ci nous avons donc su si elle avait ou non des chats, et dans l'affirmative nous avons pu en connaître le nombre et le sexe, mais aussi, pour une partie au moins d'entre eux, d'autres caractères (comme la fécondité ou l'âge) qui ne seront pas évoqués ici.

L'étude de la relation entre effectif des groupes et sexe des individus soulève une question méthodologique non négligeable que nous avons précédemment abordée à propos de populations d'insectes (LegaY \& CHESsEl, 1977). Nous avions montré alors que la constitution de couples d'individus mâle-femelle était une source de l'agrégativité spatiale, c'est-à-dire de la présence en surnombre, par rapport au modèle poissonnien, d'insectes dans certaines plantes hôtes. 
Cette question, fortement liée, au plan méthodologique, aux problèmes épidémiologiques concernant la «contagion» d'une maladie à l'échelle familiale, renvoie à trois groupes de modèles statistiques, respectivement ceux de l'analyse paramétrique des pourcentages (loi binômiale et ses dérivées) ceux de la description non paramétrique (équiprobabilité dans un espace de figures de type donné), ceux enfin des modèles log-linéaires, probit et logit (cf. Gerard \& Rolin, 1979). Ces trois types de modèles sont caractérisés par la variété des tests possibles en ce qui concerne les deux premiers, et par la complexité des modèles concevables en ce qui concerne le troisième. Nous avons choisi pour l'analyse du taux de masculinité l'approche non paramétrique.

\section{Résultats et interprétation}

\section{A. Données de base}

Toute l'information recueillie peut être rassemblée dans le tableau 1 , où sont indiqués les nombres $n_{i j}$ de familles humaines possédant un groupe de $\mathrm{i}$ chattes et $\mathrm{j}$ chats.

\section{TABleau 1}

Répartition d'un échantillon de familles humaines en fonction de l'effectif $i$ des chattes et $j$ des chats mâles présents dans le foyer.

Repartition of human families as a function of number $i$ of female cats and $j$ of male cats per family.

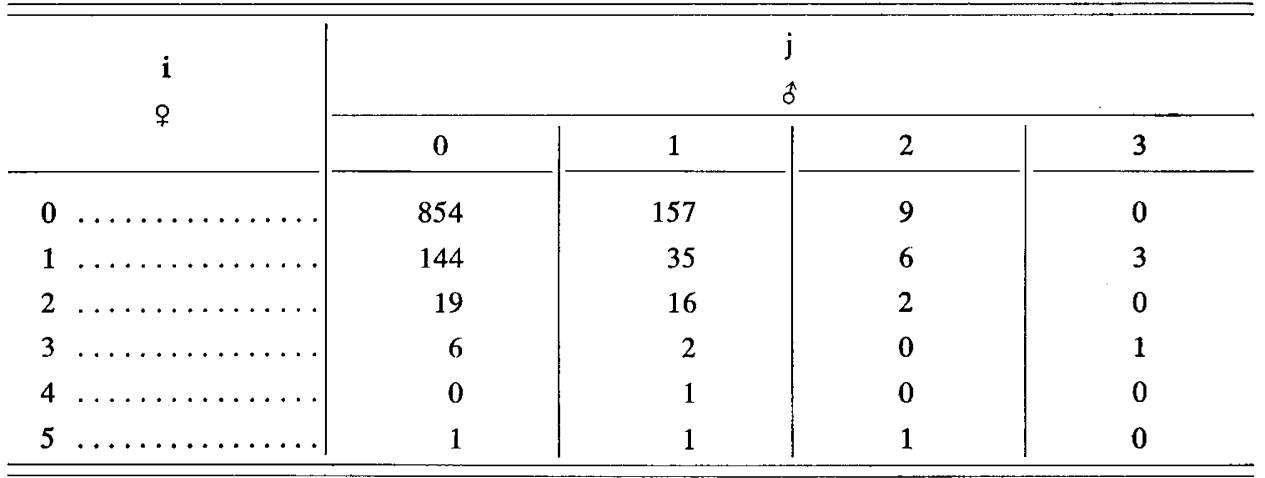

Dans cet échantillon, i varie de 0 à 5 et j de 0 à 3.568 chats ont été recensés dans les 1258 familles. Cette statistique recoupe bien, pour 17700000 foyers en France (INSEE, 1980), le nombre de chats admis pour l'ensemble du pays, soit environ 8 millions. 


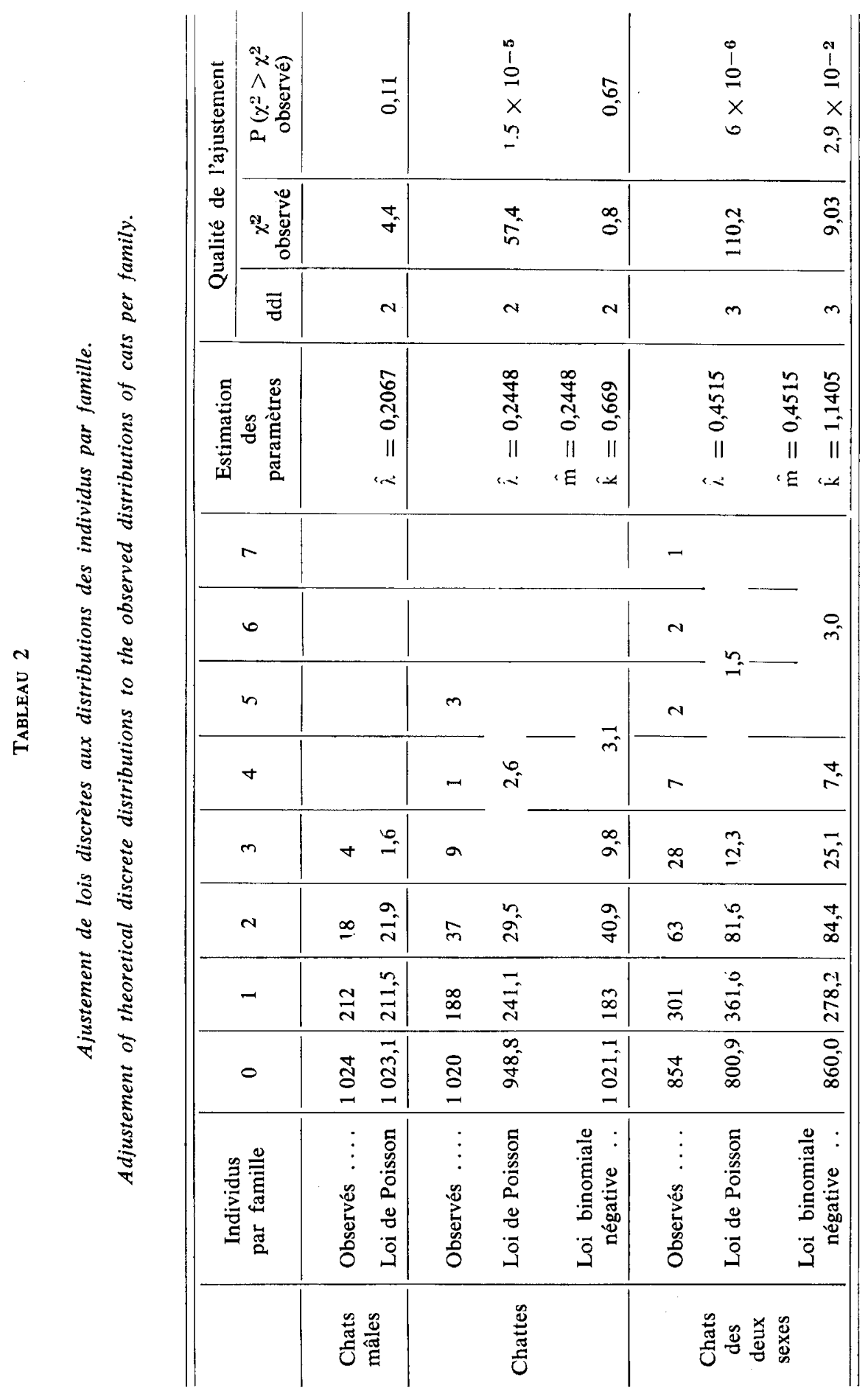




\section{B. Analyse de la répartition des individus}

L'effectif moyen des groupes est de 1,41 et celui des groupes de plus de un animal est de 2,59. Le nombre total de chats mâles égale 260 , celui des chattes égale 308. Les distributions du nombre de chats mâles, du nombre de chattes et du nombre total de chats par famille sont analysées par ajustement aux distributions discrètes les plus connues (tableau 2).

La répartition des mâles est poissonnienne, celle des femelles est binômiale négative. La répartition des individus des deux sexes ne suit pas une loi simple. A cette étape de notre étude, nous pouvons conclure à une dissymétrie mâle-femelle et à une structure complexe des groupes d'autre part.

\section{Analyse du taux de masculinité}

1. Le taux de masculinité global est-il différent de 0,5?

L'estimation

conduit au test classique :

$$
\hat{\mathrm{p}}=\frac{\mathrm{T}_{\mathrm{o}}}{\mathrm{T}}=\frac{260}{568}=0,458
$$

$$
\varepsilon=\frac{\hat{\mathrm{p}}-\mathrm{p}}{\sqrt{\mathrm{p}(1-\mathrm{p}) / \mathrm{T}}}=\frac{0,458-0,500}{\sqrt{0,25 / 568}}=-2,02
$$

valeur qui conduit au rejet de l'hypothèse nulle au niveau de signification de 5 p. 100 .

2. Le taux de masculinité dépend-il de la taille du groupe?

Pour répondre à cette question, on forme la table de contingence (tableau 3) où les chats sont rangés par sexe et par taille du groupe auquel ils appartiennent.

\section{TABleau 3}

Taux de masculinité en fonction de l'effectif des groupes.

\begin{tabular}{|c|c|c|c|c|c|c|c|c|}
\hline Effectif des groupes & 1 & 2 & 3 & 4 & 5 & 6 & 7 & Total \\
\hline $\begin{array}{l}\text { Nombre de mâles ... } \\
\text { Nombre de femelles }\end{array}$ & $\begin{array}{l}157 \\
144\end{array}$ & $\begin{array}{l}53 \\
73\end{array}$ & $\begin{array}{l}28 \\
56\end{array}$ & $\begin{array}{l}15 \\
13\end{array}$ & $\begin{array}{l}1 \\
9\end{array}$ & $\begin{array}{l}4 \\
8\end{array}$ & $\begin{array}{l}2 \\
5\end{array}$ & $\begin{array}{l}260 \\
308\end{array}$ \\
\hline Total $\ldots \ldots \ldots$ & 301 & 126 & 84 & 28 & 10 & 12 & 7 & 568 \\
\hline Pourcentage de $\hat{\sigma}$ & 0,52 & 0,42 & 0,33 & 0,54 & 0,10 & 0,33 & 0,29 & \\
\hline
\end{tabular}

Sex-ratio as a function of group size.

A cette table correspond un $\chi^{2}=18,3$ très significatif pour un degré de liberté de 6 : la fréquence des mâles n'est donc pas constante avec l'effectif du groupe.

Cette hypothèse est rejetée au niveau de signification $5 \times 10^{-3}$. 
3. Le taux de masculinité décroît-il systématiquement avec la taille du groupe?

On peut ici utiliser le test non paramétrique proposé par Gros \& CHEssEL (1982) pour comparer la taille moyenne des groupes auxquels appartiennent respectivement les mâles et les femelles. Le test porte sur la quantité

$$
\mathrm{z}=\sum_{\mathbf{i}} \mathrm{i} \cdot \mathrm{m}_{\mathrm{i}}
$$

où $\mathrm{m}_{\mathrm{i}}$ est le nombre de chats mâles dans les groupes de $\mathrm{i}$ chats et s'appuie sur la quantité

$$
\mathrm{z}_{\mathrm{o}}=\frac{\mathrm{z}-\mathrm{E}(\mathrm{z})}{\sqrt{\operatorname{Var}(\mathrm{z})}}=-3,13
$$

L'hypothèse nulle est rejetée au niveau de signification $10^{-3}$.

On en conclut que la proportion de mâles, qui évolue avec la taille du groupe, a une forte tendance à diminuer lorsque celle-ci augmente.

\section{TABLEAU 4}

Modification du taux de masculinité entre les individus solitaires et les individus vivant en groupe.

Variation of sex-ratio between isolated individuals and individuals living in groups.

\begin{tabular}{c|c|c}
\hline \hline Effectif des groupes & 1 & $>1$ \\
\hline Nombre de mâles . . . . . . & 157 & 103 \\
Nombre de femelles ....... & 144 & 164 \\
\hline \hline
\end{tabular}

Les irrégularités qui apparaissent pour les groupes de 4 chats au moins (du fait de leur fréquence de plus en plus faible) donnent l'idée de réduire la table précédente à deux modalités (tableau 4). La valeur du test $\chi^{2}=10,52$ pour 1 degré de liberté conduit à rejeter l'hypothèse nulle au seuil $10^{-3}$. Il y a donc modification du taux de masculinité dans les groupes comportant deux chats au moins, et on peut retenir, par simplification, les estimations suivantes :

$$
\begin{array}{ll}
\text { familles comportant un seul chat } & \hat{\mathbf{p}}=0,500 \\
\text { familles comportant deux chats et plus : } & \hat{\mathrm{p}}=0,386
\end{array}
$$

\section{Vérification}

On peut enfin retourner à l'étude des familles possédant deux chats au moins à l'aide du seul paramètre $\hat{\mathrm{p}}=0,386$ et du modèle d'indépendance des deux sexes dans la famille (tableau 5).

L'ajustement ( $\chi^{2}=4,23$ avec 4 degrés de liberté) n'autorise pas le rejet du modèle. Le trait porté sur le tableau 5 indique le regroupement effectué pour l'exécution du test. 


\section{Tableau 5}

Fréquence des familles observées et calculées sous l'hypothèse d'indépendance des sexes et d'un taux de masculinité de 0,386.

Observed and calculated family frequencies under the hypothesis of sex independence with $\hat{p}=0.386$.

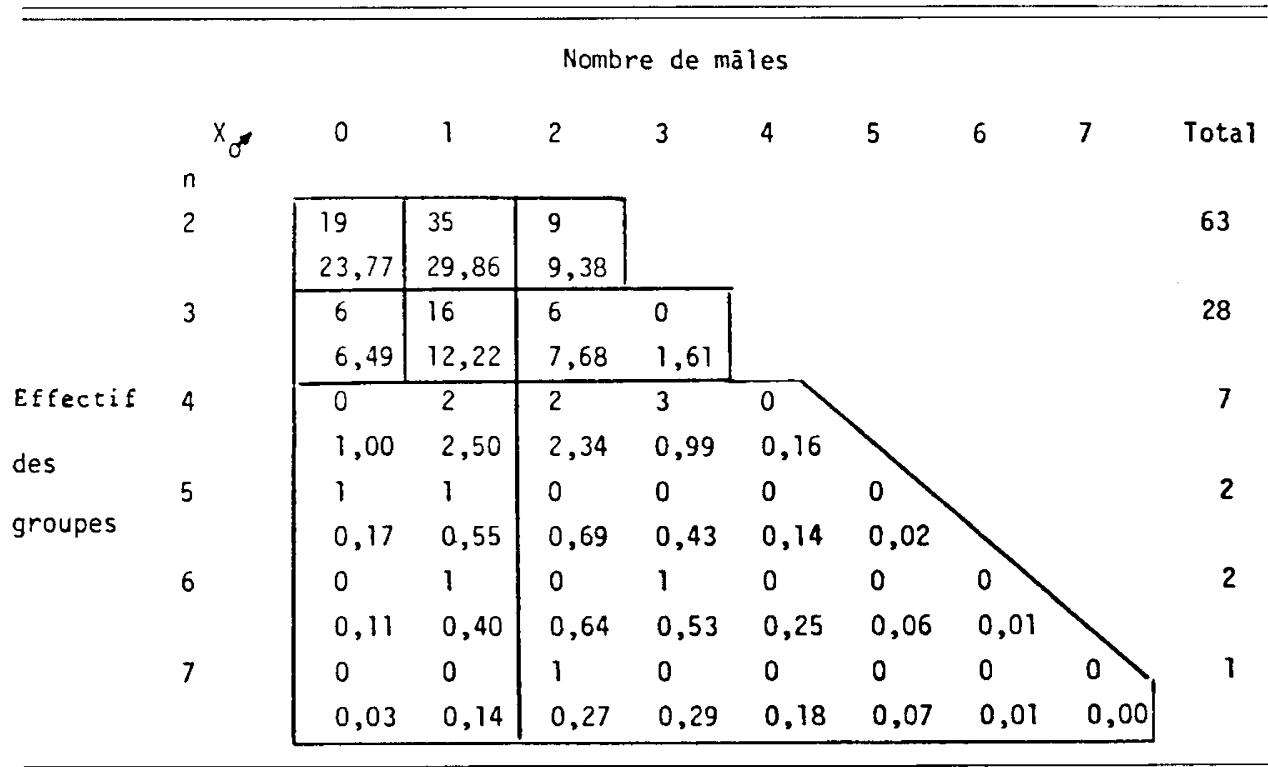

Les compartiments de ce tableau indiquent les regroupements effectués pour le calcul du $\chi^{2}$ du paragraphe 4.

The values in each compartment of the table correspond to the $\chi^{2}$ calculated as in paragraph 4.

Nous ne pouvons guère aller plus loin dans l'état actuel de nos données pour les interpréter. En effet si le taux de masculinité diminue significativement des groupes de 1 aux groupes de 3 chats, les autres groupes, d'effectif égal ou supérieur à 4 , sont en trop petit nombre (12 au total) pour qu'une analyse statistique soit fiable : en particulier le taux de masculinité des groupes de 4 n'est peut-être qu'un accident d'échantillonnage.

\section{Discussion - Conclusions}

En résumé, notre étude conduit aux résultats suivants :

- l'ordre de grandeur du nombre total de chats en France n'est pas contredit par notre échantillon, ce qui laisse supposer qu'il est assez représentatif de l'ensemble de la situation française ; 
- le nombre de familles humaines ayant au moins un chat est estimé à 32 p. 100 , pourcentage un peu plus élevé que celui que l'on avait admis jusqu'alors ;

- la distribution des chats mâles dans les familles humaines est poissonnienne ; celle des chats femelles suit une loi binômiale négative ; mais la distribution conjointe est plus complexe;

- l'analyse du taux de masculinité est par suite justifiée et révèle le caractère binômial du nombre de mâles, conditionnellement au nombre total de chats dans un groupe ; le taux de masculinité n'est pas indépendant de l'effectif du groupe;

- le pourcentage de mâles, un peu supérieur à 50 p. 100 pour l'ensemble des chats vivant seuls, baisse significativement pour les groupes de deux, puis pour les groupes de trois, mais on ne peut conclure définitivement parce que le nombre de groupes disponibles d'au moins quatre chats est trop petit.

Nous soulignerons d'abord, malgré l'importance des relations entre hommes et chats et l'intervention des uns sur les autres (surtout en milieu urbain), la liberté de manœuvre que conservent les chats d'une part dans la reproduction, d'autre part dans leur localisation spatiale et familiale. On aurait pu s'attendre à un large déséquilibre des sexes dans les familles ne possédant qu'un chat, il n'en est rien; l'influence humaine est sans doute moins directive qu'on ne pouvait le penser. Clark (1975) a même montré, pour divers quartiers de Glasgow, que la préférence humaine pour une couleur de pelage ne se traduisait pas par une sélection pour un locus quelconque. Nous accumulons de notre côté des données et des observations sur ce thème, mais il est loin d'être simple : pratiquement l'ensemble des relations homme-chat est mis en cause par cette question.

D'autre part, la comparaison de l'effectif des groupes d'après nos données et celles de VAN AARDE est intéressante. En effet l'effectif moyen pour l'ensemble des groupes est pour nous en milieu anthropisé de 1,41 , alors qu'il est seulement de 1,16 à l'île Marion, ce qui conduirait à supposer que l'homme favorise l'agrégativité, ou tout au moins la diminution du nombre des isolés. En effet l'effectif moyen des groupes d'au moins deux chats est de 2,59 dans notre échantillon et de 2,65 dans celui de VAN AARDE, ce qui est remarquablement proche pour des conditions très différentes. Mais bien entendu, nous ne voyons là que des indications, et nous devons être très prudents dans de telles comparaisons.

Le troisième commentaire concerne le fait que le nombre de mâles diminue plus vite que celui des femelles quand l'effectif des groupes augmente. Tout se passe comme si une sorte d'effet de groupe s'exerçait sur le taux de masculinité. Deux types de mécanismes semblent pouvoir expliquer ce phénomène. D'un part une forte agrégativité des femelles pourrait se traduire au moins temporairement par un excès de couples mères-filles. D'autre part, plus l'effectif des groupes augmente, plus il peut y avoir interaction avec l'âge des membres du groupe, celui-ci ne se distribuant pas forcément de la même manière selon le sexe. En outre, et de façon qualitative, on note dans les groupes de trois chats l'absence totale de groupes de trois mâles, dans les groupes de quatre chats l'absence totale de groupes de quatre femelles comme de quatre mâles. Nous n'avons pas trouvé plus de trois mâles dans un groupe, alors qu'on peut observer jusqu'à cinq femelles.

Enfin l'âge des chats des groupes a été examiné sur l'échantillon étudié dans cet article ainsi que sur quatre autres populations (LEGAY \& Pontrer, 1983). Nous avons 
montré que l'âge moyen des groupes a légèrement tendance à diminuer en fonction de leur effectif ; une mortalité différentielle entre mâles et femelles est également indiquée. La stabilité de ces résultats avec l'origine géographique des populations échantillonnées et avec l'époque des enquêtes est une question importante à laquelle seul un programme de recherches à long terme peut répondre.

L'examen de l'effectif et de la structure des groupes et la recherche de relations entre ces deux caractéristiques appelle de nouveaux modèles, des méthodes statistiques plus élaborées, et des données plus nombreuses. Les populations de chats peuvent sans doute constituer un bon matériel dans l'étude du problème de la structure des groupes comme fonction de leur effectif.

Reçu le 24 juillet 1982.

Accepté le 20 octobre 1983.

\section{Références bibliographiques}

CLARK J.M., 1975. The effects of selection and human preference on coat colour gene frequencies in urban cats. Heredity, 35, 2, 195-210.

Derenne P., Mougin J.C., 1976. Données écologiques sur les mammifères introduits de l'île aux Cochons, Archipel Crozet (48 ${ }^{\circ} 06^{\prime} ; 50^{\circ} 14^{\prime}$ E). Mammalia, 40, 21-53.

Dreux P., 1967. Gene frequencies in the cat population of Paris. J. Hered., 58, 89-92.

Dreux P., 1978. Populations rurales de chats et effet urbain. Carnivore Genet. Newsl., 3, 220-224.

FAGEN R.M., 1978. Populations structure and social behavior in the domestic cat (Felis catus). Carnivore Genet. Newsl., 3, 276-281.

Gerard G., Rolin J.M., 1979. Analyse des données discrètes, Collection Recyclage en statistique, Université catholique de Louvain.

Gros G., Chessel D., 1982. Un test exact de comparaison de deux moyennes de variables discrètes : utilisation en toxicologie. Biométrie-Praximétrie, 22, 117-130.

JoNEs E., 1977. Ecology of the feral cat, Felis catus (L.) (Carnivora Felidae) on Macquaria Island. A ust. Wildl. Res., 4, 249-262.

Legay J.M., Chessel D., 1977. Description et analyse de la répartition des insectes dans une population végétale. Cas du Doryphore sur pomme de terre. Bull. Ecol., 8, 23-34.

LegaY J.M., Pontier D., 1983. Age, courbe de survie et taille des groupes dans les populations de chats. C.R. Acad. Sci., sér. D, 296, 33-36.

Pascal M., 1980. Contribution à l'étude de la structure et de la dynamique de la population de chats harets de l'archipel Kerguelen. Thèse de doctorat de $3^{\mathbf{a}}$ cycle, Paris VI.

Robinson R., 1977. Genetics for cat breeders. Pergamon Press, London.

Robinson R., 1980. Evolution of the domestic cat. Carnivore Genet. Newsl., 4, 46-56.

Searle A.G., 1949. Gene frequencies in London's cats. J. Genet., 49, 3, 214-220.

SeARle A.G., 1968. Comparative genetics of coat colour in mammals, Logos Press, London.

Todd N.B., 1977. Les chats et les échanges commerciaux, Pour la Science, 3, 74-82.

VAN AARDE R.J., 1978. Reproduction and population ecology in the feral house cat, Felis catus, on Marion Island. Carnivore Genet. Newsl., 3, 288-316. 